\title{
Robot-Assisted Laparoscopic Nephroureterectomy versus Hand-Assisted Laparoscopic Nephroureterectomy for Upper Urinary Tract Urothelial Carcinoma: A Matched Comparison Study
}

\author{
Che-Yuan Hu, ${ }^{1}$ Cheng-Kuang Yang, ${ }^{2}$ Chao-Yuan Huang, ${ }^{1}$ Yen-Chuan Ou, ${ }^{2}$ \\ Shun-Fa Hung, ${ }^{3}$ Shiu-Dong Chung, ${ }^{3}$ and Yeong-Shiau $\mathrm{Pu}^{1}$ \\ ${ }^{1}$ National Taiwan University Hospital, No. 7, Chung Shan S. Road (Zhongshan S. Road), Zhongzheng District, \\ Taipei City 10002, Taiwan \\ ${ }^{2}$ Taichung Veterans General Hospital, Taiwan Boulevard, Sect. 4, Taichung 40705, Taiwan \\ ${ }^{3}$ Far Eastern Memorial Hospital, No. 21, Sec. 2, Nanya S. Road, Banqiao District, New Taipei City 220, Taiwan \\ Correspondence should be addressed to Chao-Yuan Huang; cyh540909@gmail.com and Yen-Chuan Ou; ycou228@gmail.com
}

Received 5 June 2015; Revised 17 July 2015; Accepted 2 August 2015

Academic Editor: Sabine D. Brookman-May

Copyright (C) 2015 Che-Yuan Hu et al. This is an open access article distributed under the Creative Commons Attribution License, which permits unrestricted use, distribution, and reproduction in any medium, provided the original work is properly cited.

\begin{abstract}
Objectives. To investigate the perioperative and oncological outcomes of hand-assisted laparoscopic nephroureterectomy (HANU) and robotic-assisted nephroureterectomy (RANU). Methods. Patients who underwent RANU were matched by sex, age ( \pm 5 years), and tumor location to those who underwent HANU; 18 matched pairs were included. Results. Each group consisted of five men and 13 women. The mean age was 70.4 years in RANU group and 69.6 years in HANU group $(p=0.646)$. Each group contained 10 patients with tumor location in the renal pelvis, five in the ureter, and three in both sites. The median follow-up time was 6.1 months for the RANU group and 47.8 months for the HANU group. The demographic and pathological data did not differ significantly. The RANU group had significantly less blood loss $(p<0.001)$, resumed oral intake earlier $(p=0.043)$, and had shorter hospital stays $(p=0.014)$ but higher pain scores associated with their wounds $(p=0.043)$. The oncological outcomes were comparable with those of the HANU group. Conclusions. Our results show that the RANU and HANU groups have comparable operative, early postoperative, and functional outcomes. A longer follow-up period would be needed for final comparison of oncological outcome.
\end{abstract}

\section{Introduction}

Since laparoscopic nephroureterectomy (LNU) was introduced in 1991 by Clayman et al. [1], it has lowered postoperative morbidity rates and shortened hospital stays compared with open nephroureterectomy [2]. The drawbacks of the laparoscopic approach are longer operative time and the requirements for high levels of laparoscopic skill, since intracorporal suturing of the bladder is often needed after bladder cuff resection. Additionally, when the tumor is located in the distal third of the ureter, it often takes considerable effort to perform pure LNU, due to the increased difficulty of bladder cuff management. In contrast, hand-assisted laparoscopic nephroureterectomy (HANU) allows the surgeon to keep one hand within the body. This approach affords the use of tactile sensation, blunt manual dissection, and broad retraction. It also decreases operative time and allows surgeons to perform minimally invasive procedures for larger and more extensive tumors $[3,4]$. Since the incidence of upper tract urothelial carcinoma (UTUC) is relatively high (about $31.4 \%$ ) in Taiwan [5] compared to all other genitourinary malignancies (about $5 \%$ for the world) [6], HANU is more often used to reduce operative time and get better control of tumors.

In 2006, the first da Vinci Robot System (Intuitive Surgical, Sunnyvale, CA, USA) was put into operation in Taiwan. With advances in the use of robotic urologic surgery, a number of medical centers in Taiwan now have experience of robot-assisted nephroureterectomy (RANU) [7]. 


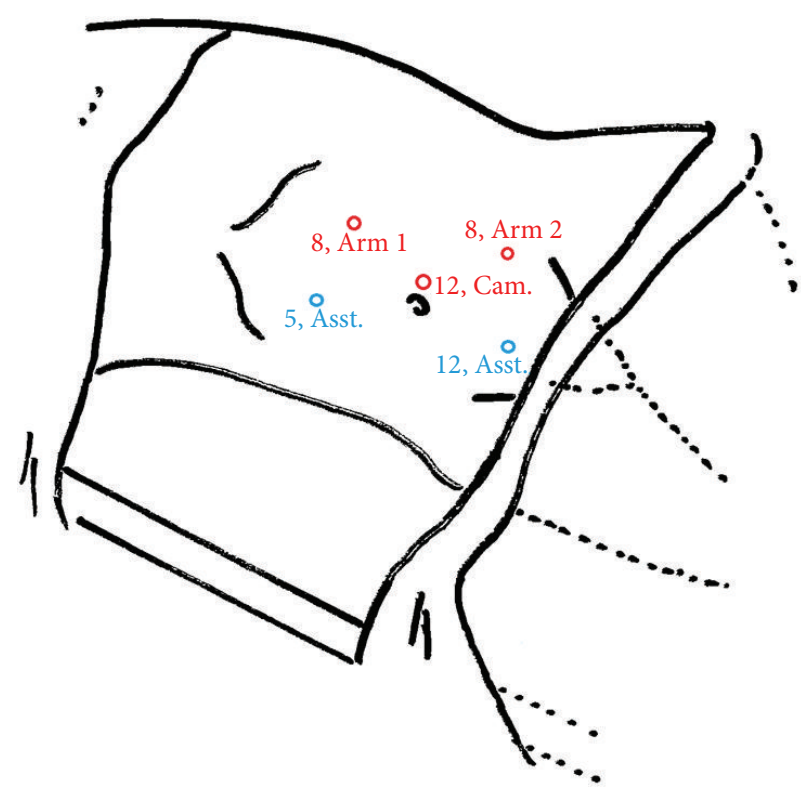

(a) Nephroureterectomy

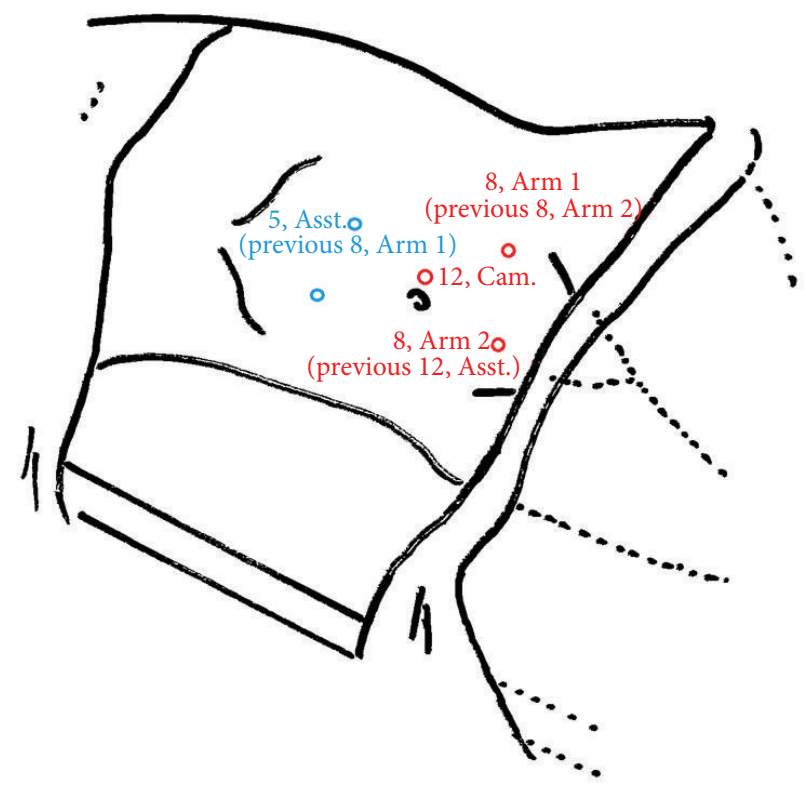

(b) Bladder cuff resection

FIGURE 1: Robot-assisted laparoscopic nephroureterectomy in left-side, upper tract urothelial carcinoma patients; right-side in the symmetric configuration. 8, Arm 1: $8 \mathrm{~mm}$ working port for robotic arm 1; 8, Arm 2: $8 \mathrm{~mm}$ working port for robotic arm 2; 5, Asst.: $5 \mathrm{~mm}$ working port for assistant; 12, Asst.: $12 \mathrm{~mm}$ working port for assistant; 12, Cam.: $12 \mathrm{~mm}$ camera port.

By reducing the difficulty of intracorporal suturing, RANU has the potential to make lower ureter and bladder cuff management easier than pure LNU. Nonetheless, the advantages and disadvantages of this surgery are not well documented, because of a lack of comparative studies between RANU and other minimally invasive techniques. We thus sought to compare the perioperative outcomes and short-term oncological outcomes of RANU and HANU.

\section{Patients and Methods}

The Institutional Review Board from National Taiwan University Hospital (NTUH) Research Ethics Committee (REC) approved this retrospective study and waived the informed consent requirement. From 2011 to 2013, eighteen UTUC patients who underwent RANU were exactly matched by sex, age ( \pm 5 years), and tumor location in a stepwise procedure to patients who underwent HANU from 2000 to 2013. Propensity score matching was not applied in this study. Excluding those that did not meet oncologic diagnosis or those who lacked sufficient data, a total of 197 patients who underwent HANU were eligible for matching. Eventually 18 matched pairs with UTUC were included in the study. Surgeons chose the operative method for UTUC patients during the time that RANU and HANU were both available. Each group consisted of five men and 13 women. In the RANU group, the patients were not repositioned after the nephrectomy. Nonetheless, the robot was redocked for excision of the distal ureter and bladder cuff.

We placed the patient in the lateral flank position with the diseased side up. A $12 \mathrm{~mm}$ camera port was then inserted in the periumbilical region and pneumoperitoneum was created. The first $8 \mathrm{~mm}$ robotic port was placed two fingers wide beneath the 12th rib, and the second $8 \mathrm{~mm}$ robotic port was introduced at the lateral edge of the rectus muscle, $3-4 \mathrm{~cm}$ below the umbilicus. A $12 \mathrm{~mm}$ assistant port was set up in the middle of the umbilicus and symphysis pubis, and a $5 \mathrm{~mm}$ assistant port was established in the middle of the umbilicus and xiphoid process.

We redocked the robot system for ureterectomy and bladder cuff resection after kidney dissection. The port for the first robotic arm became an assistant port. The port for the second robotic arm was converted to the port for the first robotic arm, and the assistant port was altered to the port for the second robotic arm [10]. We use this instrument configuration to dissect the distal ureter and bladder cuff, and intracorporeal suturing was performed to close the bladder wound (Figure 1). Finally, a $7 \mathrm{~cm}$ incision was made over lower abdominal midline near umbilicus for the specimen extraction.

In the HANU group, we made $7 \mathrm{~cm}$ Gibson's incision and created a site for the hand port, ureter identification and ligation, radical nephrectomy, and then distal ureter dissection. The remaining adventitial attachments to the bladder were identified by gentle traction on the ureter and dividing it with a dissector. Suturing of the bladder cuff and specimen extraction were accomplished through open Gibson's wound.

The follow-up schedule consisted of computed tomography examinations and bone scans at 6-12-month intervals or when clinically indicated. Cystoscopy was performed every 3 months in the first year, every 6 months in the next 2 years, and then annually. The grading and staging of the UTUC were 
performed according to the 1999 World Health Organization grade classification and the 2002 Tumor, Node, Metastasis Staging System. Clinical data, including patient survival rates and recurrence-free survival rate, bladder, renal pelvis, and ureter, were collected and analyzed. The Mann-Whitney $U$ test, chi-square test, and Fisher's exact test were used for the statistical analysis. We used the Cox proportional hazard ratio (HR) model for the univariate analyses of oncological outcomes. In all of the tests, the statistical significance was set at $p<0.05$.

\section{Results}

The median follow-up time was 6.1 months for the RANU group and 47.8 months for the HANU group. The median follow-up time for all 197 patients receiving HALNU was 41.8 months. Table 1 shows the baseline characteristics of UTUC patients who underwent RANU or HANU. Table 2 shows the clinical data from the matched cohort. The mean age of the RANU group was 70.4 years, while the mean age was 69.6 years in the HANU group. Both groups had similar clinical data, especially in terms of the previous abdominal operation history, hydronephrosis, ESRD, previous urine cytology, and simultaneous bladder cancer history.

Fifty percent or more of the tumors located in the renal pelvis in both the RANU and HANU groups (Table 3). Pathological TNM stage, grade, and lymphovascular invasion were also similar. One patient in the RANU group died from lung metastasis 6 months after surgery. The initial stage of her renal pelvic tumor was T1N0 and high grade. Three patients died of urothelial cancer in the follow-up period in the HANU group. One patient had bone metastasis (initial stage: T3N0, high grade; initial tumor location: renal pelvis and ureter) and one had recurrence in previous renal fossa (initial stage: T3N1, high grade; initial tumor location: ureter). The remaining patient had pancreatic and omental metastasis (initial stage: T3N0, high grade; initial tumor location: renal pelvis).

Table 4 provides the details of the perioperative outcomes. The mean operative time was 255.17 minutes in the RANU group and 250.17 minutes in the HANU group ( $p=$ 0.333). No patient complained of ileus after surgery. The mean blood loss, days to resuming oral intake, and length of hospital stay were significantly less in the RANU group than in the HANU group. Nonetheless, the pain scores of the associated wounds after surgery was significantly lower in the HANU group than in the RANU group.

The oncological outcomes are shown in Table 5. The relatively short follow-up time for the RANU group was due to the fact that this is a relatively new technology. Although no significant difference was noted in the oncological outcomes, two cases of recurrence in the renal fossa were noted in the HANU group.

\section{Discussion}

We compared the perioperative and oncological outcomes of two methods of modified LNU, RANU, and HANU. LNU
TABLE 1: Baseline characteristics of upper urinary tract urothelial carcinoma patients.

\begin{tabular}{|c|c|c|c|}
\hline Variable & $\begin{array}{c}\text { RANU } \\
n=18\end{array}$ & $\begin{array}{l}\text { HANU } \\
n=197\end{array}$ & $p$ value \\
\hline Age $($ mean $\pm \mathrm{SD})$ & $70.4 \pm 6.3$ & $67.7 \pm 10.6$ & 0.295 \\
\hline \multicolumn{4}{|l|}{ Gender } \\
\hline Male & $5(27.8 \%)$ & $104(52.8 \%)$ & \multirow{2}{*}{$0.050^{*}$} \\
\hline Female & $13(72.2 \%)$ & $93(47.2 \%)$ & \\
\hline $\mathrm{BMI}($ mean $\pm \mathrm{SD})$ & $23.8 \pm 3.4$ & $24.6 \pm 5.5$ & 0.688 \\
\hline \multicolumn{4}{|l|}{ CKD stage } \\
\hline Non-ESRD & $15(83.3 \%)$ & $183(92.9 \%)$ & \multirow{2}{*}{0.159} \\
\hline ESRD & $3(16.7 \%)$ & $14(7.1 \%)$ & \\
\hline Simultaneous bladder UC & $4(22.2 \%)$ & $27(13.7 \%)$ & 0.303 \\
\hline \multicolumn{4}{|l|}{ Location } \\
\hline Kidney & $10(55.6 \%)$ & $117(59.4 \%)$ & \multirow{3}{*}{0.886} \\
\hline Ureter & $5(27.8 \%)$ & $51(25.9 \%)$ & \\
\hline Both & $3(16.7 \%)$ & $29(14.7 \%)$ & \\
\hline \multicolumn{4}{|l|}{ Tumor $(\mathrm{T})$ stage } \\
\hline $\mathrm{Ta}$ & $3(16.7 \%)$ & $89(45.4 \%)$ & \multirow{5}{*}{0.052} \\
\hline $\mathrm{T} 1$ & $5(27.8 \%)$ & $30(15.3 \%)$ & \\
\hline $\mathrm{T} 2$ & $6(33.3 \%)$ & $58(29.6 \%)$ & \\
\hline $\mathrm{T} 3$ & $4(22.2 \%)$ & $16(8.2 \%)$ & \\
\hline $\mathrm{T} 4$ & 0 & $3(1.5 \%)$ & \\
\hline \multicolumn{4}{|l|}{ Node $(\mathrm{N})$ stage } \\
\hline No & $17(94.4 \%)$ & $175(93.4 \%)$ & \multirow{4}{*}{1.000} \\
\hline N1 & $1(5.56 \%)$ & $13(6.6 \%)$ & \\
\hline $\mathrm{N} 2$ & 0 & $6(3.0 \%)$ & \\
\hline N3 & 0 & $3(1.5 \%)$ & \\
\hline Metastasis (during surgery) & 0 & 0 & 1.000 \\
\hline \multicolumn{4}{|l|}{ Grade } \\
\hline Low & $1(5.56 \%)$ & $24(12.2 \%)$ & \multirow{2}{*}{0.702} \\
\hline High & $17(94.4 \%)$ & $173(87.8 \%)$ & \\
\hline
\end{tabular}

HANU: hand-assisted laparoscopic nephroureterectomy; RANU: robotassisted laparoscopic nephroureterectomy; BMI: body mass index; CKD: chronic kidney disease; UC: urothelial cancer. ${ }^{*} p<0.05$ indicates statistical significance.

provides good perioperative outcomes and cosmetic benefits [14]. HANU is used as an alternative, minimally invasive option with reliable cancer control for patients with advanced stage or tumor burdens. However, one recent study compared the outcomes of 722 patients who underwent pure LNU and 279 cases underwent HANU. It found that the use of the hand-assisted approach was associated with a higher bladder cancer recurrence rate $(p<0.01)$. The authors hypothesized that hand manipulation in the limited abdominal cavity may enhance tumor cell seeding and result in an increased intravesical recurrence rate [15]. RANU is a new technique that applies a magnified three-dimensional, highly precise vision system and tiny wristed instruments that bend and rotate like human wrists. Relatively few comparisons of the two techniques have been published showing the perioperative benefits and oncological outcomes of HANU and RANU.

In our study of these two techniques, most of the clinicopathological characteristics of the patients were very 
TABLE 2: Clinical characteristics of upper urinary tract urothelial carcinoma patients.

\begin{tabular}{|c|c|c|c|}
\hline Variable & $\begin{array}{c}\text { RANU } \\
n=18\end{array}$ & $\begin{array}{c}\text { HANU } \\
n=18\end{array}$ & $p$ value \\
\hline Age $($ mean $\pm \mathrm{SD})$ & $70.4 \pm 6.3$ & $69.6 \pm 5.7$ & 0.646 \\
\hline \multicolumn{4}{|l|}{ Gender } \\
\hline Male & $5(27.8 \%)$ & $5(27.8 \%)$ & \multirow{2}{*}{1.000} \\
\hline Female & $13(72.2 \%)$ & $13(72.2 \%)$ & \\
\hline $\mathrm{BMI}($ mean $\pm \mathrm{SD})$ & $23.8 \pm 3.4$ & $25.0 \pm 4.9$ & 0.411 \\
\hline Hydronephrosis & $12(66.7 \%)$ & $11(61.1 \%)$ & 1.000 \\
\hline Previous abdominal operation & $5(27.8 \%)$ & $4(22.2 \%)$ & 1.000 \\
\hline \multicolumn{4}{|l|}{ URS biopsy pathology } \\
\hline Negative & $6(33.3 \%)$ & $10(55.6 \%)$ & \multirow{2}{*}{0.186} \\
\hline Positive & $12(66.7 \%)$ & $8(44.4 \%)$ & \\
\hline \multicolumn{4}{|l|}{ Urine cytology } \\
\hline Negative & $13(72.2 \%)$ & $14(77.8 \%)$ & \multirow{2}{*}{1.000} \\
\hline Positive & $5(27.8 \%)$ & $4(22.2 \%)$ & \\
\hline \multicolumn{4}{|l|}{ CKD stage } \\
\hline Non-ESRD & $15(83.3 \%)$ & $16(88.9 \%)$ & \multirow{2}{*}{1.000} \\
\hline ESRD & $3(16.7 \%)$ & $2(11.1 \%)$ & \\
\hline Simultaneous bladder UC & $4(22.2 \%)$ & $4(22.2 \%)$ & 1.000 \\
\hline Herb use & $1(5.6 \%)$ & $2(11.1 \%)$ & 0.486 \\
\hline \multicolumn{4}{|l|}{ ASA class } \\
\hline I & 0 & 0 & \multirow{4}{*}{0.051} \\
\hline II & $8(44.4 \%)$ & $3(16.7 \%)$ & \\
\hline III & $6(33.3 \%)$ & $10(55.6 \%)$ & \\
\hline IV & 0 & $2(11.1 \%)$ & \\
\hline
\end{tabular}

HANU: hand-assisted laparoscopic nephroureterectomy; RANU: robotassisted laparoscopic nephroureterectomy; BMI: body mass index; URS: ureteroscopic; CKD: chronic kidney disease; ESRD: end-stage renal disease; UC: urothelial cancer; ASA: American Society of Anesthesiologists Physical Status.

similar (previous abdominal operation history, hydronephrosis before surgery, urine cytology before surgery, ESRD before surgery, and simultaneous bladder cancer history), even though we matched only the age, sex, and tumor locations. Ambani et al. [16] conducted a matched comparison of RANU $(n=22)$ and LNU $(n=22)$. They found that the mean operative time (298 versus 251 minutes) and estimated blood loss (380 versus $233 \mathrm{~mL}$ ) were significantly higher for RANU ( $p=0.03$ and $p=0.02$, resp.). They proposed that the lack of experience with robotic surgery and robotic arm repositioning were the key factors influencing the prolonged operative time. They reported a higher rate of lymph node dissection (LND, 59\% versus $27 \%$ ) in the RANU group than in the LNU group, but the application of LND did not make a difference in operative time upon further analysis. Nevertheless, the application of LND was considered a possible explanation for the greater blood loss in the RANU group. The authors proposed that the features of magnified vision and the wristed instrumentation made the surgeons more confident to perform more extensive LND. However, due to the ambiguities regarding the benefits of LND during nephroureterectomy [17], we only performed LND if no significant complication or prolonged operative
TABLE 3: Pathological characteristics of upper urinary tract urothelial carcinoma patients.

\begin{tabular}{lccc}
\hline Variable & $\begin{array}{c}\text { RANU } \\
n=18\end{array}$ & $\begin{array}{c}\text { HANU } \\
n=18\end{array}$ & $p$ value \\
\hline Location & & & \\
$\quad$ Kidney & $10(55.6 \%)$ & $10(55.6 \%)$ & \\
$\quad$ Ureter & $5(27.8 \%)$ & $5(27.8 \%)$ & 1.000 \\
$\quad$ Both & $3(16.7 \%)$ & $3(16.7 \%)$ & \\
Tumor (T) stage & & & \\
Ta & $3(16.7 \%)$ & $7(38.9 \%)$ & \\
T1 & $5(27.8 \%)$ & $2(11.1 \%)$ & 0.165 \\
T2 & $6(33.3 \%)$ & $2(11.1 \%)$ & \\
T3 & $4(22.2 \%)$ & $7(38.9 \%)$ & \\
Node (N) stage & & & \\
N0 & $17(94.4 \%)$ & $16(88.9 \%)$ & 1.000 \\
N1 & $1(5.56 \%)$ & $2(11.1 \%)$ & \\
Metastasis (during surgery) & 0 & 0 & 1.000 \\
Grade & & & \\
Low & $1(5.56 \%)$ & $3(16.7 \%)$ & 0.603 \\
High & $17(94.4 \%)$ & $15(83.3 \%)$ & \\
Lymphovascular invasion & $3(16.7 \%)$ & $4(22.2 \%)$ & 0.691 \\
\hline
\end{tabular}

HANU: hand-assisted laparoscopic nephroureterectomy; RANU: robotassisted laparoscopic nephroureterectomy.

time was expected and whenever clinically indicated. In our study, the blood loss was $68.89 \mathrm{~mL}$ in the RANU group versus $358.33 \mathrm{~mL}$ in the HANU group $(p<0.001)$.

Ambani et al. placed their patients in the lithotomy position. They redocked the robotic system from the ipsilateral side of the patients' lesion to the side between their legs to complete the bladder cuff resection, whereas we kept the robotic system on the lateral side of the patients during surgery. The operative time did not significantly differ between the RANU and the HANU groups (255.17 versus 250.17 minutes, $p=0.333$ ) in our study, in contrast to the findings of their earlier work. Park et al. [10] reported their initial hybrid-port technique for nephroureterectomy without redocking of the robot, and the total operative time was reduced by about 54 minutes compared to the method requiring relocation of the robot in between the patients' legs. The difference between the operative time in our study ( 255.17 minutes) and that of Ambani et al. (298 minutes) is about 43 minutes, which supports Park et al.s findings.

In our study, no ileus or abdominal fullness sensation was noted after nephroureterectomy in either arm of the study, but the patients in the RANU group resumed oral intake earlier than those in the HANU group $(p=0.043)$. Additionally, the length of the hospital stay was shorter in the RANU group $(p=0.014)$. Despite these benefits, patients reported greater pain scores with RANU $(p=0.043)$. Since the total length of the surgical wounds was about the same $(7 \mathrm{~cm})$ in both groups after specimen retrieval, we thought this had little contribution to the difference in pain scores. Moreover, the oblique wound (Gibson's incision) across the lower quarter of the abdomen in the HANU 
TABLE 4: Perioperative outcome of hand-assisted laparoscopic nephroureterectomy (HANU) versus robot-assisted laparoscopic nephroureterectomy (RANU).

\begin{tabular}{lccc}
\hline Variable & RANU & HANU \\
$n=18$ & 18 & $p$ value \\
\hline Operative time, min (range) & $255.17(110-540)$ & $250.17(140-410)$ & 0 \\
Ileus & 0 & $358.33(50-2000)$ & 1.000 \\
Blood loss, $\mathrm{mL}$ & $68.89(10-350)$ & $2.17(1-3)$ & $<0.001^{*}$ \\
Resumption of oral intake, days after NU & $1.59(0.5-3)$ & $9.61(4-26)$ & $0.043^{*}$ \\
Hospital stay, days & $6.79(3.7-12)$ & $3.93(3-6)$ & $0.014^{*}$ \\
Pain score, Visual Analog Scale of 1-10 & $6.22(3-10)$ & $0.043^{*}$ \\
\hline
\end{tabular}

${ }^{*} p<0.05$ indicates statistical significance.

TABLE 5: Oncological outcomes of hand-assisted laparoscopic nephroureterectomy (HANU) versus robot-assisted laparoscopic nephroureterectomy (RANU).

\begin{tabular}{|c|c|c|c|}
\hline Variable & $\begin{array}{c}\text { RANU } \\
n=18\end{array}$ & $\begin{array}{c}\text { HANU } \\
n=18\end{array}$ & $p$ value \\
\hline Median follow-up, months (range) & $6.1(0.6-30.3)$ & $47.8(11.9-156.5)$ & \\
\hline Overall recurrence & $6(33.3 \%)$ & $10(55.6 \%)$ & 0.720 \\
\hline Tumor recurrence in the bladder & $2(11.1 \%)$ & $6(33.3 \%)$ & 0.849 \\
\hline Tumor recurrence in the renal fossa & 0 & $2(11.1 \%)$ & 0.516 \\
\hline Tumor recurrence in the previous ureter site & 0 & 0 & 1.000 \\
\hline Distant recurrence (metastasis) & $4(22.2 \%)$ & $2(11.1 \%)$ & 0.093 \\
\hline Cancer-specific death & $1(5.6 \%)$ & $3(16.7 \%)$ & 0.729 \\
\hline Overall death & $2(11.1 \%)$ & $5(27.8 \%)$ & 0.781 \\
\hline
\end{tabular}

group should make those patients feel more pain because of the transection of muscle and subcutaneous nerve in the abdominal wall. Due to the relatively crude movements of the robotic arm outside of the patients' bodies, more pressure may be applied on the cutaneous wound when patients were receiving RANU. This might explain the higher pain scores in the RANU group.

Table 6 lists the OP time, blood loss, hospital stay, and complications of patients receiving RANU in other study series. Most of the researches reported their initial experience of RANU, and the results varied from study to study. The blood loss was relatively low in our case, and we ascribe this to our previous practice with regard to other robotic-assisted urological surgeries [18], which helped us to perform RANU more proficiently.

The limitation of our study is the inherent bias of the retrospective design and small sample size. In addition, the follow-up period was relatively short. Furthermore, the surgeons' and patients' preferences determined the operation methods. Therefore, the statistical significance of the results should be interpreted with caution.

\section{Conclusion}

Our results show that RANU and HANU have comparable operative, early postoperative, and functional outcomes. The RANU group is superior with regard to blood loss, resumption of oral intake, and hospital stays, although higher pain scores associated with the wounds are noted. A longer followup would be needed for final comparison of oncological outcomes, although no difference is noticed in the present series.
Abbreviations
HANU: Hand-assisted laparoscopic nephroureterectomy
RANU: Robot-assisted laparoscopic nephroureterectomy
BMI: Body mass index
URS: Ureteroscopic
CKD: Chronic kidney disease
ESRD: End-stage renal disease
UTUC: Upper tract urothelial carcinoma
ASA: American Society of Anesthesiologists Physical Status
BCR: Bladder cuff resection
OP: Operation.

\section{Conflict of Interests}

Dr. Che-Yuan Hu, Cheng-Kuang Yang, Shiu-Dong Chung, Shun-Fa Hung, Yen-Chuan Ou, Chao-Yuan Huang, and Yeong-Shiau Pu declare that they have no competing interests with regard to this work.

\section{Authors' Contribution}

Che-Yuan Hu and Cheng-Kuang Yang designed this study and collected clinical data; Che-Yuan Hu drafted this paper; 
TABLE 6: Other study series of patients receiving RANU.

\begin{tabular}{|c|c|c|}
\hline Study & Characteristics & Perioperative outcomes \\
\hline Nanigian et al. 2006 [8] & $\begin{array}{c}10 \text { patients } \\
\text { Laparoscopic NU and robotic assisted laparoscopic BCR }\end{array}$ & $\begin{array}{l}\text { (1) Mean OP time: } 264 \text { min } \\
\text { (2) Average hospital stay: } 3 \text { days }\end{array}$ \\
\hline Hu et al. 2008 [9] & $\begin{array}{c}\text { (1) Five patients } \\
\text { Flank position for NU; } \\
\text { lithotomy position for distal ureter resection and BCR } \\
\text { (2) Four patients } \\
\text { Flank position for NU and BCR }\end{array}$ & $\begin{array}{l}\text { (1) Mean blood loss: } 211 \mathrm{~mL} \\
\text { (2) Mean OP time: } 303 \mathrm{~min} \\
\text { (3) Mean hospital stay: } 2.3 \text { days }\end{array}$ \\
\hline Park et al. 2009 [10] & $\begin{array}{c}\text { (1) Six patients } \\
\text { Flank position for NU; } \\
\text { lithotomy position for distal ureter resection and BCR } \\
\text { (2) Five patients } \\
\text { Flank position for NU and BCR }\end{array}$ & $\begin{array}{l}\text { (1) Mean blood loss: } 106.7 \text { versus } 270.0 \mathrm{~mL} \\
\text { (2) Mean OP time: } 247.3 \text { versus } 193.0 \mathrm{~min} \\
\text { (3) Mean hospital stay: } 7.0 \text { versus } 8.4 \text { days } \\
\text { (4) Complication: } 0 \text { versus } 0\end{array}$ \\
\hline Eandi et al. 2010 [11] & $\begin{array}{c}\text { 11 patients } \\
\text { Flank position for NU; } \\
\text { lithotomy position for distal ureter resection and BCR }\end{array}$ & $\begin{array}{l}\text { (1) Median blood loss: } 200 \mathrm{~mL} \\
\text { (2) Median OP time: } 326 \mathrm{~min} \\
\text { (3) Mean hospital stay: } 4.7 \text { days }\end{array}$ \\
\hline Hemal et al. 2011 [12] & $\begin{array}{l}15 \text { patients } \\
\text { Flank position for NU and BCR }\end{array}$ & $\begin{array}{l}\text { (1) Mean blood loss: } 103 \mathrm{~mL} \\
\text { (2) Mean OP time: } 184 \mathrm{~min} \\
\text { (3) Mean hospital stay: } 2.7 \text { days }\end{array}$ \\
\hline Pugh et al. 2013 [13] & $\begin{array}{l}\qquad 3 \text { patients } \\
\text { Flank position for NU and BCR }\end{array}$ & $\begin{array}{l}\text { (1) Mean blood loss: } 131 \mathrm{~mL} \\
\text { (2) Mean OP time: } 247 \mathrm{~min} \\
\text { (3) Median hospital stay: } 3 \text { days }\end{array}$ \\
\hline
\end{tabular}

NU: nephroureterectomy; BCR: bladder cuff resection; OP: operation.

Shun-Fa Hung and Shiu-Dong Chung collected clinical data; Yen-Chuan Ou, Chao-Yuan Huang, and Yeong-Shiau Pu performed the operations and further paper editing. All authors read and approved the final paper. Che-Yuan $\mathrm{Hu}$ and Cheng-Kuang Yang contributed equally to this paper.

\section{Acknowledgment}

The authors would like to express their very great appreciation for the language supports provided by the National Cheng Kung University Foreign Language Center.

\section{References}

[1] R. V. Clayman, L. R. Kavoussi, R. S. Figenshau, P. S. Chandhoke, and D. M. Albala, "Laparoscopic nephroureterectomy: initial clinical case report," Journal of Laparoendoscopic Surgery, vol. 1, no. 6, pp. 343-349, 1991.

[2] J. J. Rassweiler, M. Schulze, R. Marrero, T. Frede, J. Palou Redorta, and P. Bassi, "Laparoscopic nephroureterectomy for upper urinary tract transitional cell carcinoma: is it better than open surgery?" European Urology, vol. 46, no. 6, pp. 690-697, 2004.

[3] S.-D. Chung, S.-C. Chen, S.-M. Wang et al., "Long-term outcome of hand-assisted laparoscopic nephroureterectomy for pathologic T3 upper urinary tract urothelial carcinoma," Journal of Endourology, vol. 23, no. 1, pp. 75-80, 2009.

[4] J. Landman, R. Y. Lev, S. Bhayani et al., "Comparison of hand assisted and standard laparoscopic radical nephroureterectomy for the management of localized transitional cell carcinoma," Journal of Urology, vol. 167, no. 6, pp. 2387-2391, 2002.
[5] M.-H. Yang, K.-K. Chen, C.-C. Yen et al., "Unusually high incidence of upper urinary tract urothelial carcinoma in Taiwan," Urology, vol. 59, no. 5, pp. 681-687, 2002.

[6] G. Verhoest, S. F. Shariat, T. F. Chromecki et al., "Predictive factors of recurrence and survival of upper tract urothelial carcinomas," World Journal of Urology, vol. 29, no. 4, pp. 495501, 2011.

[7] C. Y. Lin, C. R. Yang, C. L. Cheng et al., "Application in robotic urologic surgery," Journal of the Chinese Medical Association, vol. 77, no. 5, pp. 242-245, 2014.

[8] D. K. Nanigian, W. Smith, and L. M. Ellison, "Robot-assisted laparoscopic nephroureterectomy," Journal of Endourology, vol. 20, no. 7, pp. 463-466, 2006.

[9] J. C. Hu, J. P. Silletti, and S. B. Williams, "Initial experience with robot-assisted minimally-invasive nephroureterectomy," Journal of Endourology, vol. 22, no. 4, pp. 699-704, 2008.

[10] S. Y. Park, W. Jeong, W. S. Ham, W. T. Kim, and K. H. Rha, "Initial experience of robotic nephroureterectomy: a hybridport technique," BJU International, vol. 104, no. 11, pp. 1718-1721, 2009.

[11] J. A. Eandi, R. A. Nelson, T. G. Wilson, and D. Y. Josephson, "Oncologic outcomes for complete robot-assisted laparoscopic management of upper-tract transitional cell carcinoma," Journal of Endourology, vol. 24, no. 6, pp. 969-975, 2010.

[12] A. K. Hemal, I. Stansel, P. Babbar, and M. Patel, "Roboticassisted nephroureterectomy and bladder cuff excision without intraoperative repositioning," Urology, vol. 78, no. 2, pp. 357364,2011

[13] J. Pugh, S. Parekattil, D. Willis, M. Stifelman, A. Hemal, and L.-M. Su, "Perioperative outcomes of robot-assisted nephroureterectomy for upper urinary tract urothelial carcinoma: a multi-institutional series," BJU International, vol. 112, no. 4, pp. E295-E300, 2013. 
[14] S. Ni, W. Tao, Q. Chen et al., "Laparoscopic versus open nephroureterectomy for the treatment of upper urinary tract urothelial carcinoma: a systematic review and cumulative analysis of comparative studies," European Urology, vol. 61, no. 6, pp. 1142-1153, 2012.

[15] M. Z. Aslam and M. Agarwal, "Detrusor myectomy: long-term functional outcomes," International Journal of Urology, vol. 19, no. 12, pp. 1099-1102, 2012.

[16] S. N. Ambani, A. Z. Weizer, J. S. Wolf Jr., C. He, D. C. Miller, and J. S. Montgomery, "Matched comparison of robotic vs laparoscopic nephroureterectomy: an initial experience," Urology, vol. 83, no. 2, pp. 345-349, 2014.

[17] G. Lughezzani, C. Jeldres, H. Isbarn et al., "A critical appraisal of the value of lymph node dissection at nephroureterectomy for upper tract urothelial carcinoma," Urology, vol. 75, no. 1, pp. $118-124,2010$.

[18] Y.-C. Ou, C.-K. Yang, K.-S. Chang et al., "The surgical learning curve for robotic-assisted laparoscopic radical prostatectomy: experience of a single surgeon with 500 cases in Taiwan, China," Asian Journal of Andrology, vol. 16, no. 5, pp. 728-734, 2014. 


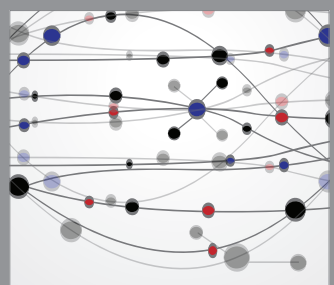

The Scientific World Journal
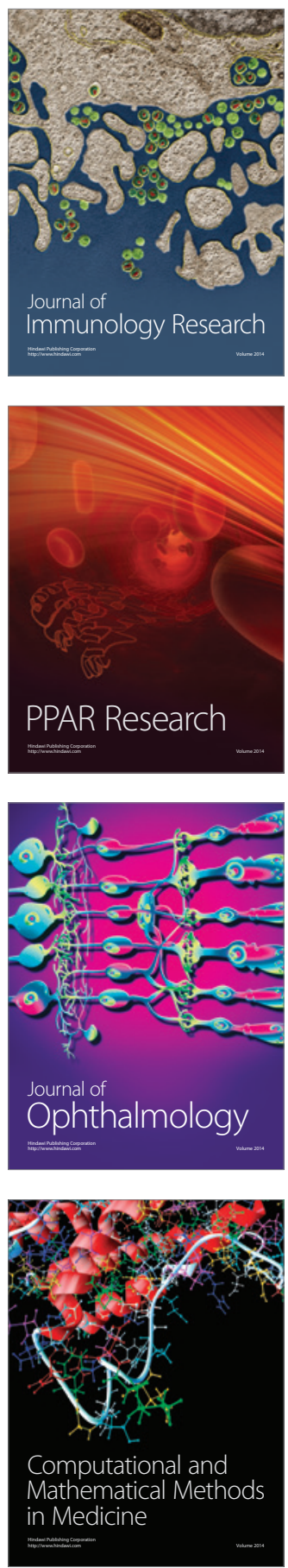

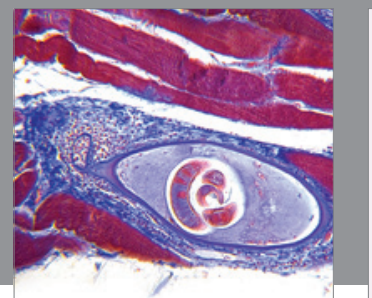

Gastroenterology

Research and Practice
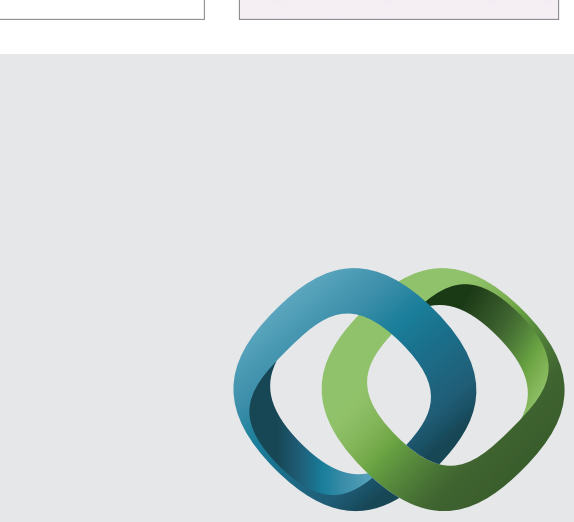

\section{Hindawi}

Submit your manuscripts at

http://www.hindawi.com
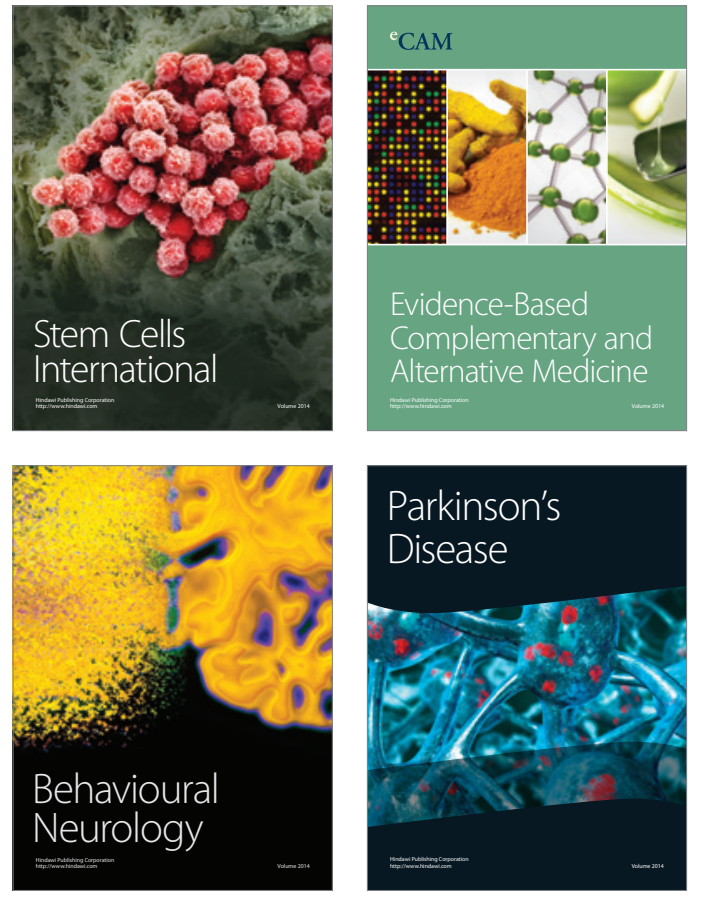
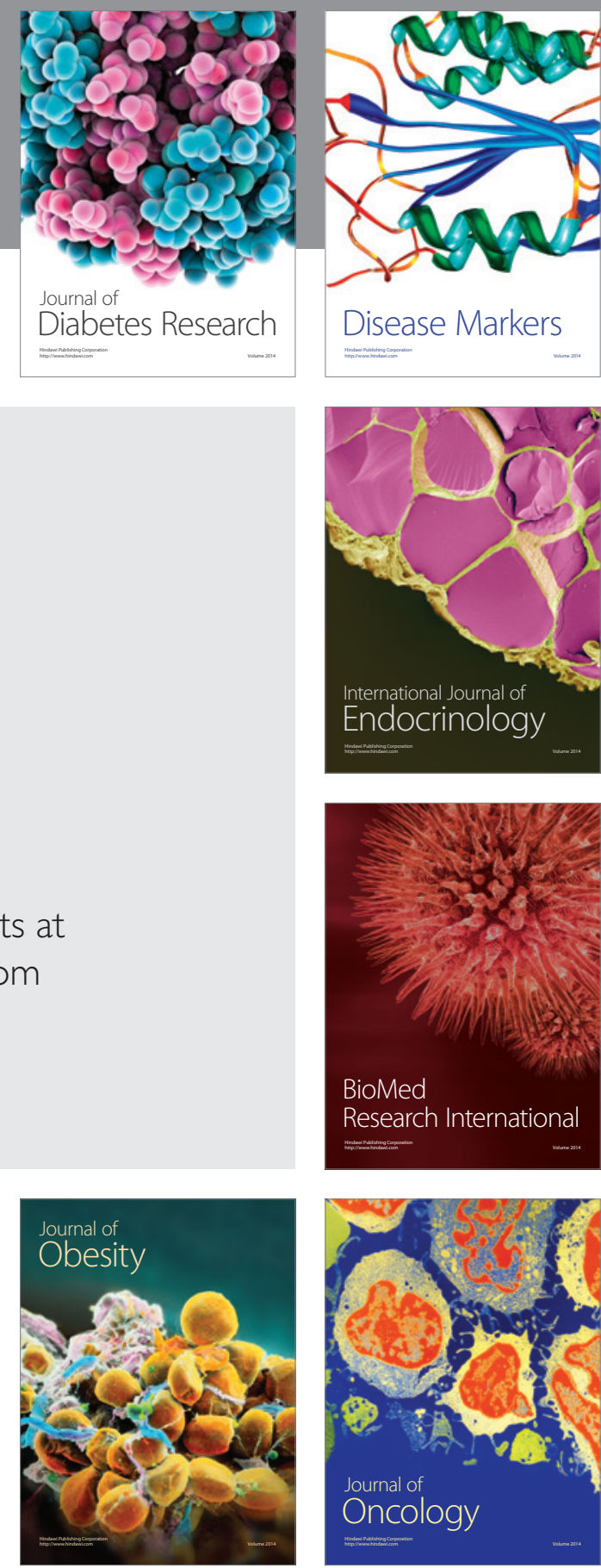

Disease Markers
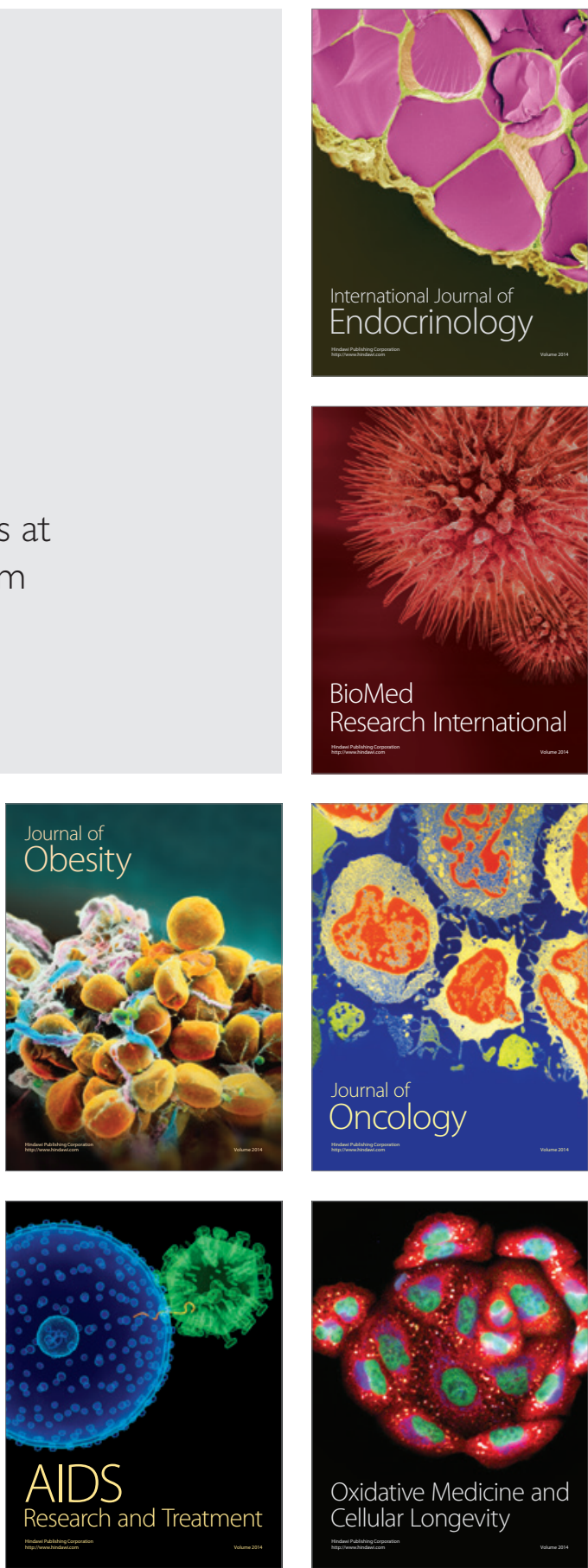